\title{
Characteristics of Patients Sponsored by the Internet Platforms of Medical Mutual Aid
}

\author{
Xiaotong Li, Feng Qian \\ Department of Physiology, School of Basic Medicine, Health Science Center, Yangtze University, Jingzhou, China \\ Email: 49605795@qq.com
}

How to cite this paper: Li, X.T. and Qian, F. (2021) Characteristics of Patients Sponsored by the Internet Platforms of Medical Mutual Aid. Yangtze Medicine, 5, 70-78. https://doi.org/10.4236/ym.2021.51008

Received: June 2, 2020

Accepted: January 112021

Published: January 14, 2021

Copyright $\odot 2021$ by author(s) and Scientific Research Publishing Inc. This work is licensed under the Creative Commons Attribution International License (CC BY 4.0).

http://creativecommons.org/licenses/by/4.0/ (c) (i) Open Access

\begin{abstract}
The morbidity and mortality of life-threatening diseases such as cancers are increasing year by year in China. Although the national medical insurance has been improved a lot, it still falls short of expecting "universal medical insurance". To help the families of patients with cancer and other serious diseases to resolve the unaffordable medical cost, several internet platforms of "Peer to Peer Medical Mutual Aid" have gradually emerged recently. In the form of low threshold insurance combined with public support, these platforms provide subsidies to families of patients suffering from serious financial burden and then partly compensate them for the limit of national medical insurance. This article summarized the characteristics of declared patients sponsored by several medical mutual aid internet platforms. The results showed that the sponsored patients were mostly cancer patients (90.1\%), aged $21-60(88.4 \%)$, and the average amounts of financial support had a trend to decrease with the increase of age. In particular, the reported cases of thyroid cancer were increased dramatically recently, however, with a lowest average mutual aid fund. Given these data, it is urgent to list out a detailed rule for the use of national medical insurance on the treatment of cancer, to heighten the healthcare system for the occupational population, age from 21 to 60 , and to establish the credit system for Medicare and Medicaid services.
\end{abstract}

\section{Keywords}

Medical Insurance, Cancer, Thyroid Cancer, Internet Platform

\section{Introduction}

Statistical analysis shows that in 2015, in China the incidence of malignant tumors was about $0.29 \%$ and the mortality rate of malignant tumors was about $0.17 \%$ [1]. About the economic load of serious disease treatment, cancer is the 
main disease causing poverty in low- and middle-income families [2]. Although the participation rate of national basic medical insurance has reached above $95 \%$, and the "universal medical insurance" has taken early shape, there are still many deficiencies that need to be improved [3]. The internet platform of medical mutual aid originated from the internet public welfare platform for serious diseases. In the early stage, the main way of internet medical financial support is launching crowd funding to provide certain economic support for the families of poor patients. However, after the internet mutual aid platform became increasingly popular, fraud incidents emerged one after another because of the lack of official supervision of such public welfare and standardization for clarifying the poverty of receivers [4]. It results in the loss of public enthusiasm for such public welfare crowd funding. Referring to relevant foreign cases, the peer to peer internet platforms of medical mutual aid have gradually developed into an extra form of peer to peer medical insurance with a low cost [5]. But its specific role in improving the national medical insurance remains to be seen. Therefore, collecting and analyzing the population characteristics of the funded patients published by these network platforms are conducive to assess the reliability and need of these platforms for low- and middle-income families. It's also valuable to analyze the deficiencies of the national basic medical insurance system and to find the weakness in guarantee mechanism of peer to peer mutual aid platforms.

\section{Materials and Methods}

Declared peer to peer medical mutual aid fund sponsored cases were collected from various online platforms of medical mutual aid from August 2018 to July 2019. The collected cases were further screened by identifying the personal information and clinical characteristics of the funded patients. Qualified cases should contain the following details: the patient's name, gender, age, place of origin, diagnosis certificate, location of hospitalization, treatment status and the amount of received mutual aid fund. The cases with incomplete or inaccurate information were excluded. Sociodemographic and clinical characteristics of the funded patients were compared and analyzed to provide reasonable suggestions for improving the medical insurance system and the medical security system.

Statistical analyses were conducted by using SPSS version 21.0 (Statistical Product and Service Solutions, Chicago, IL, USA). The characteristics distributions of male and female patients were assessed by the Chi square test. One way ANOVA and post hoc test were applied to analyze the variance among different factors. A $P$ value $<0.05$ was considered significant. The mean values are presented as Mean \pm SE.

\section{Results}

This survey totally selected 3023 qualified cases of funded patients published on the internet platforms of peer to peer medical mutual aid. The types of funded diseases were mainly cancer, cardiovascular disease and cerebrovascular disease, 
accounting for $90.1 \%, 3.9 \%$ and $0.4 \%$ respectively. Among them, there were 1428 cases of male funded patients, with tumors accounting for $84.5 \%$, mainly gastrointestinal cancer, lung cancer and thyroid cancer. There were 1595 cases of female funded patients, with tumors accounting for $95.2 \%$, mainly breast cancer, uterine cancer and thyroid cancer. There were significant differences between male and female patients in the types of diseases, the ranges of patient age $(\mathrm{P}<$ $0.01)$, and the mutual aid fund $(P<0.05$, Table 1 and Figure $1(a))$. In addition,

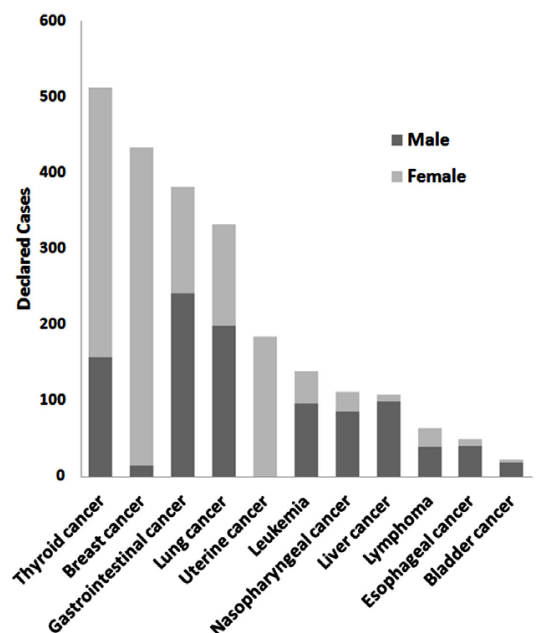

(a)

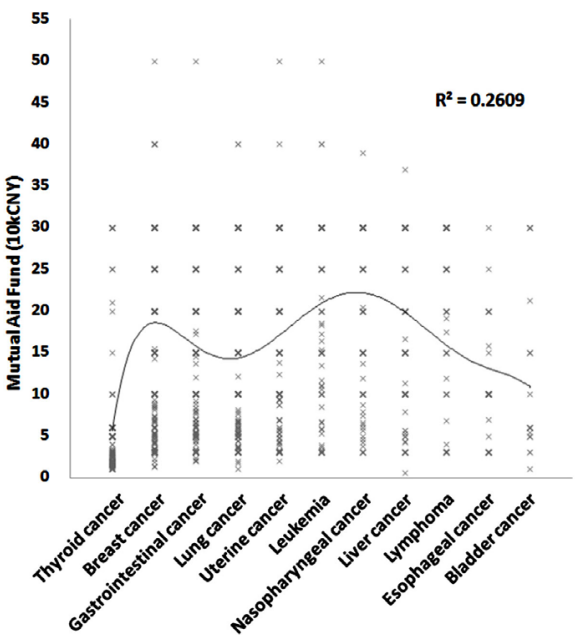

(b)

Figure 1. The declared cases (a) and mutual aid fund (b) of different cancer types. In the declared cases of sponsored patients, the thyroid cancer patients were supported with highest number but lowest average mutual aid fund $(\mathrm{P}<0.05)$.

Table 1. Clinical characteristics of the patients sponsored by medical mutual aid internet platforms.

\begin{tabular}{ccccc}
\hline & $\begin{array}{c}\text { Male (\%) } \\
\mathbf{n}=1428\end{array}$ & $\begin{array}{c}\text { Female (\%) } \\
\mathbf{n}=1595\end{array}$ & Chi-square & P \\
\hline Reason & & & 103.658 & $<0.001$ \\
Cancers & $1207(84.5 \%)$ & $1518(95.2 \%)$ & & \\
Cardiovascular accidents & $99(6.9 \%)$ & $19(1.2 \%)$ & & \\
Cerebrovascular accidents & $10(0.7 \%)$ & $4(0.2 \%)$ & & \\
Others & $112(7.9 \%)$ & $54(3.4 \%)$ & & \\
Age & & & & \\
$0-20$ & $65(4.6 \%)$ & $49(3.1 \%)$ & & \\
$20-40$ & $547(38.3 \%)$ & $742(46.5 \%)$ & & \\
$40-60$ & $670(46.9 \%)$ & $714(44.8 \%)$ & & \\
$>60$ & $146(10.2 \%)$ & $90(5.6 \%)$ & & \\
Aid fund (10 k RMB) & & & \\
$<5$ & $253(17.7 \%)$ & $223(14.0 \%)$ & & \\
$5-20$ & $823(57.6 \%)$ & $937(58.7 \%)$ & & \\
$>20$ & $352(24.7 \%)$ & $435(27.3 \%)$ & & \\
\hline
\end{tabular}


the sponsored female patients were mainly suffered from cancers and aged from 20 to 60 , especially the percentage of female patients aged from 20 to 40 is significantly higher than that of male patients $(\mathrm{P}<0.01$, Table 1$)$. Thyroid cancer accounted for the highest proportion (18.9\%) of declared cases, followed by breast cancer (15.9\%), gastrointestinal cancer (14.0\%) and lung cancer (12.2\%). The histogram of individual mutual aid fund received by each cancer patient shows that the mutual aid fund sponsored from internet platforms has a top limit set according to the requirement of recipients (Figure 1(b)). Nevertheless, the average mutual fund sponsored to these thyroid cancer patients was significantly lower than other cancer patients $(\mathrm{P}<0.05$, Figure $1(\mathrm{~b}))$.

Supported patients were mostly suffering from cancers and aged from 20 to 60, especially female patients. According to the analysis of age ladder (Table 1), all age groups had the same main reason, cancer, for mutual aid fund application. Cancer was the most common disease that caused poverty because of the heaviest economic burden from the expensive medical cost. Most of the applicants were family breadwinners aged between 21 and 60, accounting for $88.4 \%$ of all applicants. The applicants aged less than 20 and more than 60 only accounted for $3.8 \%$ and $7.8 \%$ respectively. The histograph based on the relationship of mutual aid fund and age showed that the average mutual aid fund had a weak linear correlation to decrease with the increase of age $\left(R^{2}=0.1605\right.$, Figure 2).

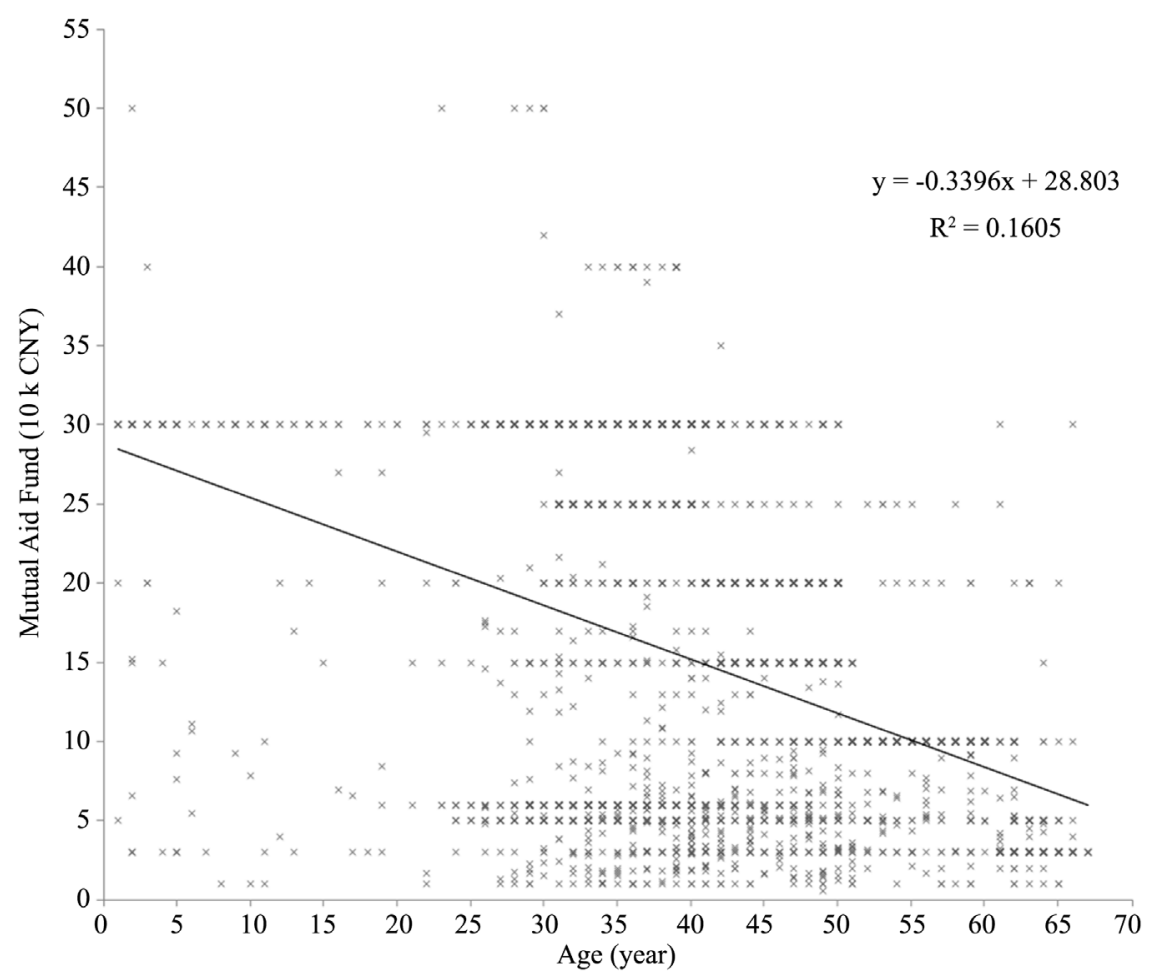

Figure 2. The histograph of medical mutual aid fund along with the age of sponsored patients. Linear regression implicated that the average mutual aid fund declined with the increase of age $\left(R^{2}=0.1605\right)$. 
Guangdong province has the highest proportion of recipients $(10.9 \%)$, followed by Shandong (10.3\%), Hunan (7.7\%), Jiangsu (7.1\%) and Sichuan (6.5\%). Notably, 98.2\% applicants from Guangdong province were cancer patients. However, considering the population base of each province, these data cannot demonstrate the differences among the places of origin of those sponsored patients.

\section{Discussion}

The internet platforms of medical mutual aid referred to the form of peer to peer mutual insurance abroad, and in recent years they began to carry out the membership system. After becoming a member by paying a little money in advance, share to afford other members' medical mutual aid fund cost to keep membership. The member suffering from serious disease or accident can apply for the mutual aid fund after the supervision and confirmation from platforms. Registering a peer to peer mutual aid member is much cheaper than those commercial medical insurance and there is no conflict between the items in the mutual aid platform and the traditional insurance [5]. Therefore, it can be used as a supplementary Medicaid system to the basic medical insurance and commercial medical insurance. The mutual aid platform is based on the Internet, and its risk control mechanism is still not perfect. It lacks enough supervision on the corroborating membership application and application data of mutual aid funds for serious diseases, and there exists moral hazard. To strengthen the supervision system, some platforms have done the publicity system, hoping to ensure the reasonable and legal use of mutual aid fund for serious diseases through public supervision [4] [5]. They declare recently sponsored cases on the internet platforms for public review. We collected these data to analyze the clinical characteristics of the patients sponsored by the peer to peer medical mutual aid internet platforms.

The average age of cancer patients funded by network mutual aid platform is significantly lower than the national level [1]. The family breadwinners, aging from 21 to 60 , account for $88.4 \%$ of the total applicants. It is quite different from the morbidity of cancer which increases with age, even for those older than 60 [1]. The proper explanation should be that the financial burden of a family is mainly borne by the disability of labor population. However, in these ages, the proportion of female grantees is higher than that of male, especially the proportion of women aged between 21 and 60 with cancer is as high as $91.3 \%(\mathrm{P}<0.01$, Table 1). The age gradient data showed the number of cancer funded patients increased significantly from the age of 28 , peaked at 38 , and the number of cancer patients applying for mutual aid began to decline after the age of 46 (Figure 2). 28 - 46 years old is the golden period for masses of employment meanwhile bearing the family economic expenses. When they suffer from serious diseases, their family's economic income is stagnant, and they are faced with expensive medical costs. Therefore, the illness of labors is easier to cause the poverty of 
their families. The subsidy amount of mutual aid fund decreased with the increase of age (Figure 2) which implicates the will of public population, to pay more health resources on younger people.

According to a survey by the poverty relief office of the state council at the end of 2015, 42\% of the poor families in China suffered from poverty due to illness. And the 2018 statistical bulletin on the development of national basic medical security, by the end of 2018, the number of people joining in the national basic medical insurance had reached 1.34 billion, and the coverage of the insurance was stable at more than $95 \%$. The number of people joining in the basic medical insurance for employees is about 320 million (Above data obtained from China government official website). The universal medical insurance system has taken shape initially. However, it still has limited protection for the insured and their families because of the uneven distribution of medical resources and defects in the medical treatment system [6]. In particular, the labors have high risk of accidents and their family may face serious economic burden if they are disabled by the diseases [6]. Therefore, the national basic medical security should be more inclined to these breadwinners and improve the medical security of them to avoid illness-induced poverty of the their families. Meanwhile, the adjustment of the treatment based on the factors such as their family population structure, local medical service resources, and the increase of medical expenses, should also be taken into full account.

The insufficient amount of medical insurance fund has been the main factor restricting its development. Under the current situation, the main direction to solve the current dilemma is improving the medical insurance policy and ensuring the legitimate and reasonable use of the medical insurance fund [6] [7]. As mentioned above, the increasing incidence of cancer has become a serious burden on the country, society and the patient's family because of its unaffordable medical cost, long treatment time, poor efficacy and poor quality of life after treatment [6]. Taking thyroid cancer as an example, it accounts for about 5.03\% of the incidence of all malignancies in China [1]. However, it accounts the highest proportion for about $18.9 \%$ of those declared cancer cases (Figure 1(a)), even higher than the proportions of breast cancer and lung cancer. It seems that the thyroid cancer patients are more likely to obtain financial supports from those peer to peer mutual aid funds. Besides, there is still considerable controversy over its diagnosis and treatment, which is in danger of being excessive medical treatment [8]. Although relevant medical experts have formulated relevant rules for the diagnosis and treatment of thyroid cancer [9], the relevant regulations for the implementation of medical insurance do not specifically consider the actual clinical situation. It is still difficult to avoid the occurrence of over-diagnosis and over-treatment in clinical practice [10]. Under this situation, the medical cost of thyroid cancer treatment is not covered by the medical insurance reasonably according to the clinical diagnosis and treatment [11]. Nevertheless, the average mutual aid fund sponsored to these thyroid cancer patients was significantly lower than other cancer patients $(\mathrm{P}<0.05$, Figure $1(\mathrm{~b}))$, be- 
cause the average medical cost of thyroid cancer should be much lower than those of other cancers. In the short of total basic medical insurance fund, still putting much fund into the medical security of cancer and other serious diseases is no doubt to overwhelm the existing financing difficulty of national Medicaid system.

The internet platforms of peer to peer medical mutual aid is a kind of public welfare supplement besides the main role of national medical insurance [5]. The data about the origin provinces of sponsored patients showed that these mutual aid platforms have been widely accepted all around the country. According to the results, the medical mutual aid fund is not enough to guarantee the basic medical service needs of the whole people. It only provides subsidies to families suffering from cancer and other serious diseases with an average amount of less than 140,000 CNY (Table 1 and Figure 2). Among the patients funded by mutual aid platforms, the proportion of cancer diseases is as high as $90.1 \%$. It indirectly shows that the unaffordable expenses on cancer treatment are the most difficult risk to be guaranteed and controlled by the current national medical insurance policy. At present, the repayment ratio of major diseases in the medical insurance policy is determined according to the rules and regulations. But there is no clear criterion to determine the severity of serious diseases such as cancer, because of the lack of clear legal definition. Poor families may give up treatment for curable diseases because they cannot afford Non-Medicare expenses. Therefore, according to the medical development, the detailed regulations on defining medical insurance for cancer treatment can be formulated. Under the premise of proper consideration of medical ethics and full respect for social equality, it will help to realize the social welfare value of medical insurance fund. A combined usage of different Medicaid systems should be better in solving this financial dilemma. The national basic medical insurance guarantees the equal access to basic medical services for all the people, the commercial medical insurance guarantees the serious diseases such as cancer, and the peer to peer mutual aid platform provides public welfare as the social supplement for the medical security of serious diseases.

In addition, whether it is the national health insurance, commercial health insurance, or network mutual assistance platform, there are fraudulent insurance, fraud, and other dishonest phenomena. In particular, the internet platforms of medical mutual aid lack enough judicial supervision and are vulnerable to the threat of moral hazard [4]. This problem should be well resolved by developing a credit system for insured individuals, medical service units, and medical insurance management units [12]. Therefore, promoting to build the medical insurance credit system is the key step to improve medical insurance at all levels and the medical public welfare [4] [5].

\section{Conclusion}

The medical cost on cancer treatment is the main economic burden of medicare and medicaid system. The internet platforms of peer to peer medical mutual aid 
can work as a public welfare supplement in constructing the national medical insurance system. Since the unreasonable distribution of insurance fund expense on cancer treatment, especially on the treatment of thyroid cancer, it is urgent to list out a detailed rule for the use of national medical insurance on the treatment of cancer. The results also arose the need to heighten the healthcare system for the labors aging from 21 to 60 .

\section{Acknowledgements}

This work was supported by grants from the Scientific Research Foundation of the Hubei Provincial Department of Health and Family Planning (WJ2016-Y-01) and the Jingzhou Science and Technology Department (2019EC61-17) to QF, and the College Students Innovative Entrepreneurial Training Program of Yangtze University to LXT.

\section{Conflicts of Interest}

The authors declare no conflicts of interest regarding the publication of this paper.

\section{References}

[1] Zheng, R.S., Sun, K.X., Zhang, S.W., et al. (2019) Report of Cancer Epidemiology in China, 2015. Chinese Journal of Oncology, 41, 19-28.

[2] Sun, D., Cao, M., Li, H., He, S. and Chen, W. (2020) Cancer Burden and Trends in China: A Review and Comparison with Japan and South Korea. Chinese Journal of Cancer Research, 32, 129-139.

[3] Pan, H. and Li, D.R. (2019) Analysis of Application Status of Medical Insurance Intelligent Audit System in China. Chinese Hospital Management, 39, 68-69. https://doi.org/10.21147/j.issn.1000-9604.2020.02.01

[4] Yang, Y. and Xiao, J. (2019) Research on Medical Insurance Fund Fraud and Anti-Fraud. Journal of Beijing University of Aeronautics and Astronautics, 32, 41-51.

[5] Yang, C. and Chen, B. (2018) A Research on P2P Insurance Pricing Based on Cooperative Game. Insurance Studies, No. 5, 33-44.

[6] Witte, J., Mehlis, K., Surmann, B., et al. (2019) Methods for Measuring Financial Toxicity after Cancer Diagnosis and Treatment: A Systematic Review and Its Implications. Annals of Oncology, 30, 1061-1070. https://doi.org/10.1093/annonc/mdz140

[7] Gan, T., Sinner, H.F., Walling, S.C., et al. (2019) Impact of the Affordable Care Act on Colorectal Cancer Screening, Incidence, and Survival in Kentucky. Journal of the American College of Surgeons, 228, 342-353.e1. https://doi.org/10.1016/j.jamcollsurg.2018.12.035

[8] Welch, H.G. and Doherty, G.M. (2018) Saving Thyroids: Overtreatment of Small Papillary Cancers. The New England Journal of Medicine, 379, 310-312. https://doi.org/10.1056/NEJMp1804426

[9] Craig, S.J., Bysice, A.M., Nakoneshny, S.C., Pasieka, J.L. and Chandarana, S.P. (2020) The Identification of Intraoperative Risk Factors Can Reduce, but Not Exclude, the Need for Completion Thyroidectomy in Low-Risk Papillary Thyroid Cancer Patients. Thyroid, 30, 222-228. https://doi.org/10.1089/thy.2019.0274 
[10] Decallonne, B., Van den Bruel, A., Macq, G., Elaut, N. and De Schutter, H. (2020) The Impact of Regional Variation in Clinical Practice on Thyroid Cancer Diagnosis: A National Population-Based Study. European Thyroid Journal, 9, 32-39. https://doi.org/10.1159/000504046

[11] Liu, Y., Wang, Y., Zhao, K., et al. (2020) Lymph Node Metastasis in Young and Middle-Aged Papillary Thyroid Carcinoma Patients: A SEER-Based Cohort Study. BMC Cancer, 20, 181. https://doi.org/10.1186/s12885-020-6675-0

[12] Bao, H. and Liu, R. (2019) Exploration on the Path of Establishing Medical Insurance Doctors' Credit Management Mechanism in Ningxia. China Health Insurance, No. $4,42-46$. 